\title{
Noncollinear magnetism in freestanding and supported monatomic Mn chains
}

\author{
Franziska Schubert, ${ }^{1, *}$ Yuriy Mokrousov, ${ }^{2, \dagger}$ Paolo Ferriani, ${ }^{3}$ and Stefan Heinze ${ }^{3}$ \\ ${ }^{1}$ Institute of Applied Physics, University of Hamburg, D-20355 Hamburg, Germany \\ ${ }^{2}$ Peter Grünberg Institut and Institute for Advanced Simulation, Forschungszentrum Jülich and JARA, D-52425 Jülich, Germany \\ ${ }^{3}$ Institute of Theoretical Physics and Astrophysics, Christian-Albrechts-University of Kiel, D-24098 Kiel, Germany
}

(Received 15 December 2010; revised manuscript received 24 February 2011; published 27 April 2011)

\begin{abstract}
Using first-principles calculations, we study the occurrence of noncollinear magnetic order in monatomic Mn chains. First, we focus on freestanding Mn chains and demonstrate that they exhibit a pronounced noncollinear ground state in a large range of interatomic distances between atoms in the chain. By artificially varying the atomic number of Mn we investigate how the magnetic ground state is influenced by alloying the Mn chains with Fe and Cr. With increasing number of $3 d$ electrons we find a smooth transition in the magnetic phase space starting from an antiferromagnetic state for pure $\mathrm{Cr}$ chains through a regime of noncollinear ground states for Mn-rich chains to a ferromagnetic solution approaching the limit of pure Fe chains. Second, we investigate the magnetism in supported $\mathrm{Mn}$ chains on the (110) surfaces of $\mathrm{Cu}, \mathrm{Pd}$, and $\mathrm{Ag}$. We show that even a weak chain-surface hybridization is sufficient to dramatically change the magnetic coupling in the chain. Nevertheless, while we observe that Mn chains are antiferromagnetic on $\operatorname{Pd}(110)$, a weak noncollinear magnetic order survives for Mn chains on $\mathrm{Cu}(110)$ and $\mathrm{Ag}(110)$ a few $\mathrm{meV}$ in energy below the antiferromagnetic solution. We explain the sensitive dependence of the exchange interaction in Mn chains on the interatomic distance, chemical composition, and their environment based on the competition between the ferromagnetic double exchange and the antiferromagnetic kinetic exchange mechanism. Finally, we perform simulations which predict that the noncollinear magnetic order of $\mathrm{Mn}$ chains on $\mathrm{Cu}(110)$ and $\mathrm{Ag}(110)$ could be experimentally verified by spin-polarized scanning tunneling microscopy.
\end{abstract}

DOI: 10.1103/PhysRevB.83.165442

PACS number(s): 73.20.-r, 71.70.Gm, 75.75.-c

\section{INTRODUCTION}

In pursuit of atomic-scale magnetic storage devices and future spintronics applications, one-dimensional (1D) transitionmetal (TM) nanostructures have become a topic of intense research interest. ${ }^{1-4}$ A promising route for future applications concerns, for example, the control of the spin state in 1D wires using spin-polarized currents via the spin transfer torque. Besides the strong technological motivation, from the fundamental point of view 1D systems constitute a unique playground for electronic structure theory. Due to enhanced intra-atomic exchange and lowered coordination, they reveal an increased tendency toward magnetism. For example, elements such as $\mathrm{Pt}$ and $\mathrm{Pd}$, which are nonmagnetic in bulk, can become magnetic in atomic chains. ${ }^{5,6}$ Moreover, giant values of the magnetic anisotropy energy, which is crucial to stabilize magnetism against thermal fluctuations, have been found for free-standing, suspended, and deposited TM chains. ${ }^{1,5,7}$ Owing to the rich physics in 1D systems many more novel effects and phenomena have been reported in the recent years concerning their electronic structure, spin dynamics, and transport properties. ${ }^{3,8-13}$

The creation of monatomic chains in experiment is extremely challenging; however, in the most recent years, a few techniques to achieve this goal have been successfully developed. One experimental approach is the formation of chains in so-called mechanically controllable break junctions. Upon pulling two electrodes apart, it is possible to produce short freestanding monatomic chains suspended between the electrodes. With this technique, successful chain formation has been reported for late $4 d$ and $5 d$ TM elements and transport measurements can be performed to probe the junction properties. ${ }^{14}$ Another route is to use a substrate and grow the chains by self-assembly exploiting 1D structures provided by the surface topography such as step edges ${ }^{1}$ or in trenches of reconstructed surfaces, as has been demonstrated for $\mathrm{Fe}$ chains on $\operatorname{Ir}(001)^{15}$ or $\mathrm{Au}$ chains on $\mathrm{Si}(111) .{ }^{16}$ A second possibility on a surface is to build the chain atom by atom utilizing a manipulation technique with the tip of a scanning tunneling microscope (STM). The use of STM is particularly attractive as it also allows for a direct study of the magnetic properties of individual chains. For example, in combination with spin-polarized STM, Serrate et al. used this approach to demonstrate noncollinear spin alignment in small linear chains of Co atoms on $\mathrm{Mn} / \mathrm{W}(110) .{ }^{4}$ On the other hand, Hirjibehedin and coworkers created linear Mn chains of up to ten atoms on an insulating $\mathrm{CuN} / \mathrm{Cu}(001)$ surface and applied inelastic STM to prove their antiferromagnetic exchange coupling. ${ }^{2}$

One of the basic issues in chain magnetism is the sign, origin, and dependence on various external parameters of the exchange interaction between the magnetic moments of the TM atoms. The most common way to model a magnetic chain of atoms is the effective Heisenberg Hamiltonian:

$$
H=-\sum_{n \neq m} J_{n m} \mathbf{S}_{n} \cdot \mathbf{S}_{m},
$$

which describes the magnetic ground state and low-energy magnetic excitations of a system from the knowledge of the Heisenberg parameters, or exchange constants, $J_{n m}$. In the latter relation $\mathbf{S}_{n}$ and $\mathbf{S}_{m}$ are the unit vectors of the spins of atoms $n$ and $m$; that is, their magnitude does not depend on their relative orientation within this model. In case of linear equidistant monatomic chains of atoms of the same kind, the Heisenberg model can be rewritten with respect to the atom at the origin with the Heisenberg constants $J_{n} \equiv J_{n 0}$. The general 
solution of the Heisenberg model on a periodic lattice, here a $1 \mathrm{D}$ monatomic chain, is the so-called (flat) spin-spiral state, which is a noncollinear arrangement of spins given by

$$
\mathbf{S}_{n}=[\cos (n d q), \sin (n d q), 0],
$$

where $d$ is the spacing between atoms in the chain and $q$ is the modulus of the spin-spiral vector. For each set of exchange parameters, the energy dispersion $E(q)$ of a spin-spiral with a wave-vector $q$, propagating along the chain axis $z$, can be determined from Eq. (1):

$$
E(q)=-2 \sum_{n} J_{n} \cos (n d q),
$$

and the ground state of the system can be found among collinear ferromagnetic (FM, $q=0$ ), collinear antiferromagnetic (AFM, $q=0.5 \times 2 \pi / d$ ), and noncollinear spin-spiral states. The phase diagram for the 1D-Heisenberg model and characteristic shapes of the curves $E(q)$ are presented in Fig. 1, where the exchange interaction was assumed to vanish beyond third-nearest neighbors.

As can be seen from Fig. 1, the phase space of possible ground states of a 1D spin chain is rather complicated and depends sensitively on the exchange parameters, exhibiting wide regions of preferred noncollinear solutions. As far as real TM chains are concerned, establishing their magnetic ground-state position in the phase diagram of Fig. 1 based on their electronic structure determined by ab initio calculations is a nontrivial task. While the exchange interactions have been explored intensively for finite TM magnetic clusters, both freestanding and deposited on surfaces, ${ }^{9,17-20}$ the work for infinite monatomic TM chains concentrated almost exclusively on collinear magnetic solutions..$^{10,21-26}$ The occurrence of noncollinear magnetic states and analysis of exchange interaction beyond the first neighbor in freestanding and deposited monatomic chains has come into the focus of interest only recently, ${ }^{27-29}$ while a conclusive evidence for a spin-spiral ground state, or more complicated spin textures,
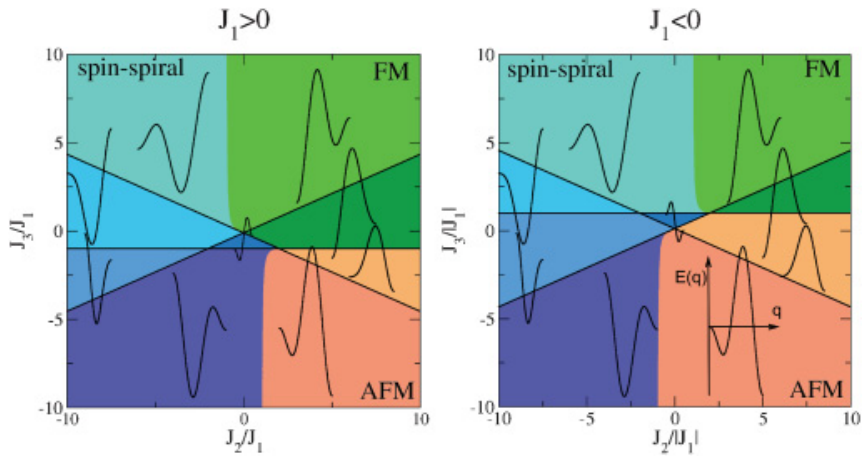

FIG. 1. (Color online) Magnetic phase diagram of the 1DHeisenberg model in the $\left(J_{2}, J_{3}\right)$-parameter space for $J_{1}>0$ (left graph) and $J_{1}<0$ (right graph). The illustrations depict the shape of the spin-spiral dispersion curves $E(q)$ in the corresponding phases, with the curve starting at $q=0$ (FM state) and ending at $q=0.5$ (AFM state) in units of $2 \pi / d$, where $d$ is the interatomic distance. The $E$ and $q$ axes have been added for one curve for clarity (lower right corner of right graph). In that particular case, the global energy minimum is at the end; that is, for $q=0.5$ and the AFM state is most favorable. in a real deposited metallic TM monatomic chain or wire with complex atomic relaxations is missing from $a b$ initio theory. Experimentally, it is very challenging to measure the exchange interaction. Nevertheless, the sign of the exchange interaction has been indirectly determined by STM for Mn, $\mathrm{Fe}$, and $\mathrm{Co}$ dimers on $\mathrm{NiAl}(110)^{30}$ and even quantitatively for the interaction between single Co atoms on $\operatorname{Pt}(111)^{31,32}$ and for $\mathrm{Mn}$ chains on $\mathrm{CuN} / \mathrm{Cu}(001){ }^{2}$ In the two latter cases, indirect exchange by the RKKY interaction and superexchange via the substrate atoms, respectively, were concluded as the microscopic mechanisms. Surprisingly, for the Mn dimer on $\mathrm{NiAl}(110) \mathrm{FM}$ coupling due to double exchange was reported. ${ }^{30}$

Among other $3 d$ TMs, Mn chains are most likely to display a manifold of magnetic solutions depending on the details of chain geometry, environment, and hybridization with the substrate. This stems from the fact that a monowire (MW) of $\mathrm{Mn}$ atoms experiences a FM-to-AFM ground-state transition as a function of the interatomic distance $d$ not far away from the equilibrium value $d_{0} \cdot{ }^{21}$ The origin of this transition lies in the subtle competition of AFM kinetic exchange and FM double exchange, ${ }^{33}$ which in terms of the $d$-electron exchange splitting $\Delta$ and hopping $t$ can be quantitatively described as a competition between the $t^{2} / \Delta$ and the $t$ terms, respectively. ${ }^{18}$ The kinetic exchange arises from a level repulsion between occupied majority states of an atom with unoccupied minority states of a neighboring atom, when the spins of the two atoms are opposite. On the other hand, the hopping between the $d$ states of the neighboring atoms gives rise to an energy gain due to the splitting between the bonding and antibonding minority $d$ states, if the spins are oriented in parallel, while this splitting is zero in the AFM configuration.

Taking a "prototypical" TM monatomic chain, we normally observe smaller values of $\Delta$ and larger values of $t$ for smaller interatomic distance $d$, which promotes the AFM kinetic exchange over the FM double exchange. Upon increasing the interatomic distance, on the other hand, the hopping $t$ is decreased while the exchange splitting energy $\Delta$ increases reaching the atomic limit value, which promotes the double exchange over the kinetic exchange, and the chain can become FM. This results in the Bethe-Slater behavior of the first nearest-neighbor exchange parameter $J_{1}$ for TM chains as a function of interatomic distance: $J_{1}$ is negative for small $d$ and becomes positive for larger $d .^{21}$ The position of this transition point, where $J_{1}$ changes sign, depends, of course, on the TM and is situated close to the equilibrium interatomic distance only for Mn chains among all $3 d$ TMs. $^{21}$

Here we investigate the appearance of noncollinear magnetism in freestanding monatomic Mn chains, its development upon alloying the chains with the neighbors of $\mathrm{Mn}$ in the periodic table, that is, $\mathrm{Cr}$ and $\mathrm{Fe}$, and finally upon deposition on the $\mathrm{Cu}(110), \operatorname{Pd}(110)$, and $\mathrm{Ag}(110)$ surfaces. Due to the special position of Mn chains at the borderline between competing FM and AFM exchange interactions, we anticipate the possibility of a spin-spiral ground state in the vicinity of this transition point, where $J_{1}$ is small, and long-ranged indirect exchange interactions become of importance. ${ }^{34}$ Indeed, a whole set of noncollinear solutions has been recently predicted to occur in single-strand as well as thicker infinite and finite freestanding wires of $\mathrm{Mn}$ atoms, ${ }^{27,29}$ revealing a sensitive dependence of 
the exchange interactions on the details of the surroundings, observed also for small deposited Mn clusters. ${ }^{18}$ Also in two dimensions the tendency of $\mathrm{Mn}$ to noncollinear spin ordering is well-established. ${ }^{35-38}$ While experimentally finite $\mathrm{Mn}$ chains on $\mathrm{CuN}$ are believed to display an AFM ordering due to superexchange via substrate atoms, a possibility for noncollinear spin ordering has been suggested for long finite Mn chains on $\mathrm{Ni}(111)$ from $a b$ initio calculations due to frustration of competing exchange interactions within the chain and with the surface. ${ }^{9}$ However, structural relaxations, which can strongly influence the magnetic order, were not considered in that study.

In our work, we demonstrate that indeed the spin-spiral solution is the ground state of freestanding Mn chains in a wide region of interatomic distance $d$ between 4.3 and 5.5 a.u. Furthermore, we consider freestanding Mn chains alloyed with $\mathrm{Cr}$ and $\mathrm{Fe}$ atoms and find that such chains take a smooth trajectory in the magnetic phase space as a function of $\mathrm{Cr}, \mathrm{Mn}$, and Fe concentration. Starting from an AFM state for $\mathrm{Cr}$ chains the region of noncollinear solutions is crossed for Mn-rich chains until with increasing Fe content the FM part of the phase diagram is reached. This suggests a simple way to tune the magnetic ground state of Mn-containing chains and wires. One can explain this transition within the Bethe-Slater ${ }^{21}$ as well as Moriya picture of direct exchange between $d$ states. ${ }^{39}$ We also investigate whether the noncollinear ground state survives for Mn chains on a substrate and consider the (110) surfaces of $\mathrm{Cu}, \mathrm{Pd}$, and $\mathrm{Ag}$ including structural relaxations. Our choice of substrates allows us to study the effect of varying interatomic distance in the chains as well as the dependence on the strength of hybridization with the substrate. We show that owing to the spin-dependent charge transfer between the chain and the surface and reduction of the exchange splitting $\Delta$ the exchange interaction changes in favor of AFM kinetic exchange, and for Mn chains on Pd(110) the ground state is AFM. On the other hand, a shallow spin-spiral ground state in the vicinity of the AFM solution is retained for Mn chains on $\mathrm{Ag}(110)$ and $\mathrm{Cu}(110)$. Since these surfaces are suitable for chain creation by self-assembly or by manipulation with an STM tip, we simulate STM experiments based on the calculated vacuum charge and magnetization density of states. We show that the spin spiral magnetic order in supported Mn MWs can theoretically be observed in a spin-polarized STM measurement allowing an unambiguous proof of the noncollinear spin structure.

\section{COMPUTATIONAL DETAILS}

In our first-principles investigation of noncollinear magnetism in monatomic Mn chains based on density-functional theory (DFT) we neglect spin-orbit coupling, which affects the electronic structure, spin, and orbital moments rather weakly for $3 d$ TM chains in general, and Mn chains in particular, due to their half-filled $d$ shell. ${ }^{24}$ Since we also focus on the metal substrates $\mathrm{Cu}, \mathrm{Ag}$, and $\mathrm{Pd}$ with relatively small spin-orbit coupling this remains a good approximation throughout our study. Neglecting the spin-orbit interaction allows for an enormous speed-up of the calculations as we need to consider only one TM-atom per unit cell due to the validity of the generalized Bloch theorem. ${ }^{40,41}$ For all calculations we used the generalized gradient approximation
rev-PBE exchange-correlation functional. ${ }^{42}$ Calculations for the freestanding MWs have been performed using the 1D full-potential linearized augmented plane-wave method as implemented in the Jülich DFT code FLEUR. ${ }^{43}$ We used between 64 and $320 k$ points carefully checking the convergence of the obtained values with respect to their number. The calculation of the spin-spiral energy dispersions was done with the magnetic force theorem, and we tested its result versus the self-consistent calculations, finding generally very good agreement. For the basis-functions cutoff we used the $k_{\max }$ parameter of more than 4.0 a.u. ${ }^{-1}$, while we chose values of 6 and 7.5 a.u. for the vacuum parameters $D_{\text {vac }}$ and $\tilde{D}$, respectively. We used a muffin-tin radius of 2.1 a.u. for $\mathrm{Mn}$ atoms.

Calculations of the surface-deposited monatomic $\mathrm{Mn}$ chains were performed with the film version of the FLEUR code $^{44,45}$ with the geometrical setup and computational details close to those in Ref. 21. We modeled the semi-infinite crystal by a slab of seven substrate layers for the fcc (110) surfaces of $\mathrm{Cu}, \mathrm{Pd}$, and $\mathrm{Ag}$. We used the experimental lattice constants of the substrates and exploited inversion symmetry by depositing chains on both sides of the slab. We restricted structural relaxations to the FM case. The following values for the muffin-tin radii were used: 2.2 a.u. for $\mathrm{Cu}$ atoms, 2.3 a.u. for $\mathrm{Pd}$ and $\mathrm{Ag}$ atoms, and 2.2 a.u. for the chain $\mathrm{Mn}$ atoms. We considered an in-plane separation between the chains of approximately 15 a.u., which corresponds to a $p(2 \times 1)$ unit cell (see Fig. 2 for a sketch of the geometrical setup). This choice of the supercell provides a separation between adjacent chains large enough to exclude the effect of interchain interaction on the spin-spiral energies and energy scales, discussed in the paper. We chose values of $k_{\max }=3.6$ to $3.8{\mathrm{a} . \mathrm{u}^{-1}}^{-1}$ (depending on the surface) for relaxations and noncollinear calculations, achieving convergence in the values of the total energy differences and spin moments. Further computational details, values of the spin moments and relaxed atomic positions can be found in Ref. 21. For the spin-spiral calculations, we considered a slab of five substrate layers to simulate the semi-infinite crystal and a dense mesh of $336 k$ points in one half of the Brillouin zone. The calculations of

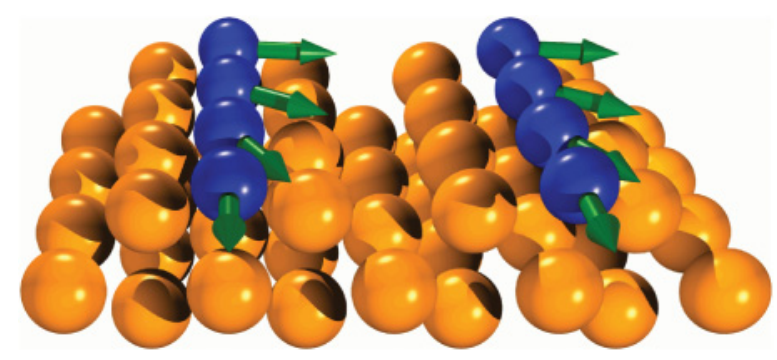

FIG. 2. (Color online) Geometrical setup for a Mn chain with interatomic distance $d$ deposited on an unreconstructed fcc (110) surface. The blue spheres represent chain atoms with arrows indicating the directions of the spin moments, while the gold spheres represent substrate atoms. The chain atoms are located in the hollow sites of the surface layer above the subsurface layer atoms. The 2D unit cell has $p(2 \times 1)$ geometry. A spin spiral state with a value of $q=0.07 \times 2 \pi / d$ has been chosen for illustration. Note that in this picture the spin spiral rotates within the film plane, however, as we neglect spin-orbit coupling the rotation plane is arbitrary. 

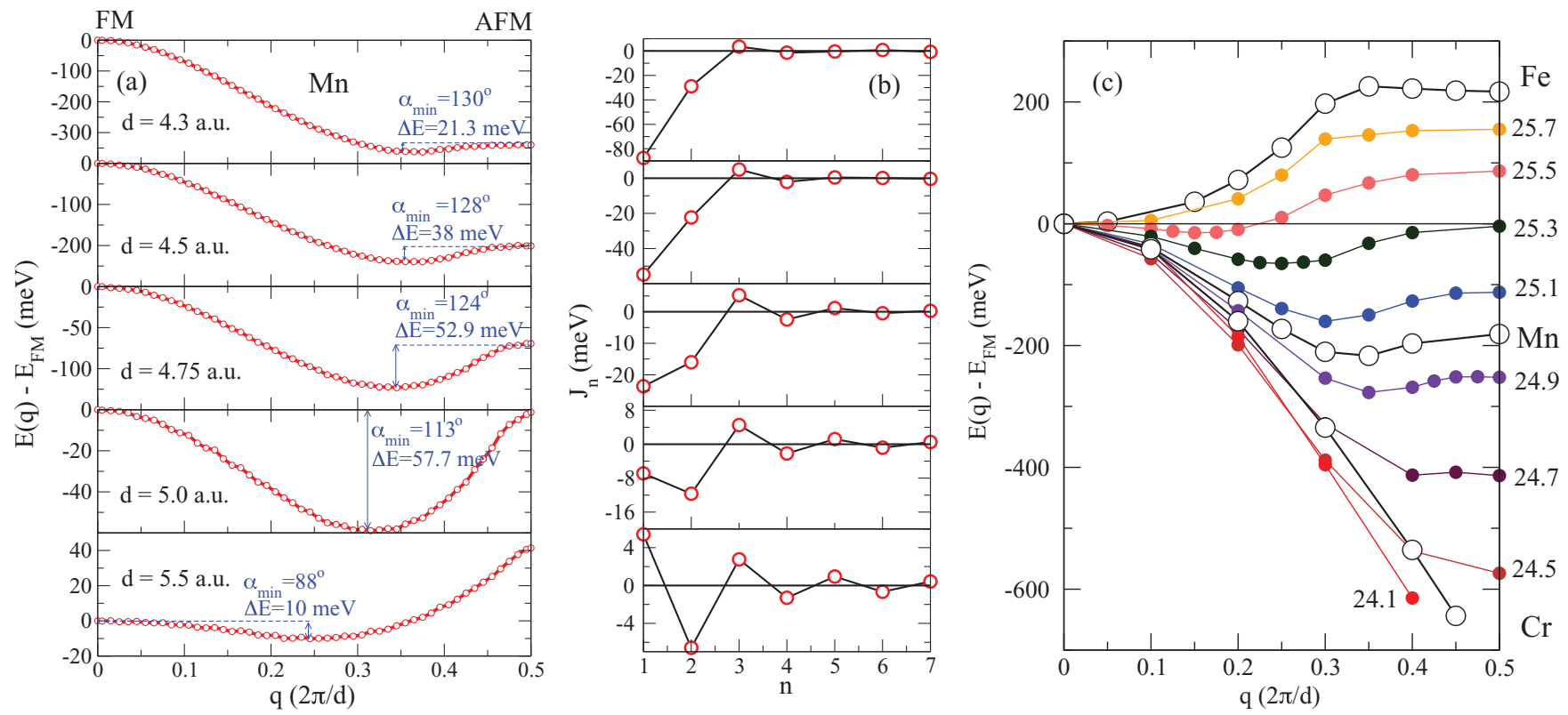

FIG. 3. (Color online) (a) Flat spin-spiral dispersions $E(q)$ for freestanding monatomic Mn chains as a function of interatomic distance $d$, calculated using the force theorem. The angle $\alpha_{\min }$ between spins of nearest-neighbor Mn atoms at the spiral minimum is given, as well as the depth $\Delta E$ of the energy minimum. (b) Corresponding Heisenberg exchange constants $J_{n}$. (c) Flat spin-spiral dispersion $E(q)$ for freestanding chains with an interatomic distance $d=4.5$ a.u. and atomic number varying between $\operatorname{Cr}(Z=24.0)$ and $\mathrm{Fe}(Z=26.0)$ calculated self-consistently. In (a) and (c) the energies are given relative to the FM state.

the spin-spiral dispersion curves for deposited Mn chains were performed self-consistently.

\section{NONCOLLINEAR MAGNETISM IN FREE-STANDING Mn CHAINS}

It was predicted from first-principles calculations ${ }^{21}$ that freestanding Mn MWs exhibit a FM-to-AFM ground-state transition as a function of interatomic distance $d$, a phenomenon that has been later predicted to occur also in $\mathrm{Mn}$ dimers. ${ }^{20}$ Experimentally, FM coupling in Mn dimers on $\mathrm{NiAl}(110)$ was deduced indirectly from tunneling spectra in STM experiments. ${ }^{30}$ According to the Heisenberg model, in the vicinity of the transition point from FM-to-AFM coupling, the nearest-neighbor Heisenberg exchange parameter, $J_{1}$, becomes comparatively small, and exchange constants beyond nearest neighbors become of importance. This can result in a pronounced noncollinear ground state of Mn chains (cf. phase diagrams in Fig. 1). Indeed, the appearance of a spin-spiral energy minimum has been recently demonstrated by ab initio calculations for free-standing chains as well as more complex structures of $\mathrm{Mn}$ atoms. ${ }^{27,29}$ In our work, we investigate how the noncollinear magnetism in Mn chains evolves upon stretching the chain and alloying with strongly AFM Cr and $\mathrm{FM} \mathrm{Fe},{ }^{21}$ alloys that are known to exist in bulk $\alpha-\mathrm{Mn}$ (see, e.g., Ref. 46).

We start by studying the magnetism in pure Mn chains in the regime of the interatomic distance $d$ around the FM-AFM crossover point between 4.3 and 5.5 a.u. ${ }^{21}$ When the spacing is varied in this interval, the spin moment of the Mn atoms changes by roughly $0.3 \mu_{B}$ with respect to the averaged value of $4 \mu_{B}$ for both FM and AFM solutions. For each value of $d$ the variation of the Mn spin moment as a function of the spin-spiral vector $q$ is even smaller (up to $3 \%$ ), which allows a mapping to the Heisenberg model. In Fig. 3(a) we present the energy dispersion relation $E(q)$ of spin spirals [cf. Eq. (3)] for $\mathrm{Mn}$ chains with different interatomic spacings $d$. The energies were evaluated with respect to the FM solution, $q=0$ ( $\Gamma$ point), while $q=0.5 \times 2 \pi / d$ ( $X$ point) corresponds to the AFM state.

Starting already at $d=4.3$ a.u. a very shallow spin-spiral energy minimum of $\Delta E=21.3 \mathrm{meV}$ with respect to the AFM state develops at $q \approx 0.35 \times 2 \pi / d$, corresponding to an angle of approximately $130^{\circ}$ between spins of nearest-neighbor $\mathrm{Mn}$ atoms. As the interatomic distance increases, this energy minimum becomes deeper, reaching as much as $57.7 \mathrm{meV}$ for a distance of 5.0 a.u., and moves toward the middle of the BZ with the angle between the spins on neighboring atoms, $\alpha_{\min }$, becoming smaller. At this distance, the difference in energy between the FM and the AFM state, negative for smaller $d$, nearly vanishes, and upon further increasing $d$, when the FM state moves to lower energies with respect to the AFM solution, the noncollinear ground-state energy minimum becomes less pronounced again. Remarkably, in the whole range of considered interatomic distance the ground state of the Mn chains is noncollinear.

In Fig. 3(b) we display the Heisenberg exchange constants $J_{n}$ as a function of the $n$ th-nearest neighbor in the chain extracted from the corresponding $E(q)$ dispersion curves in Fig. 3(a) by a Fourier transform. At a lattice constant of 4.3 a.u. $J_{1}$ is large and negative, indicating AFM exchange coupling that dominates over all other Heisenberg constants, which can be considered negligible beyond the second-nearest neighbor. Among the collinear solutions the AFM state is therefore favored over the FM state by a large value of $340 \mathrm{meV}$ and the spin-spiral minimum is shallow. With increasing $d$ 
the long-range exchange interaction becomes more significant as $J_{1}$ decreases drastically until it changes its sign to FM coupling between 5.0 and 5.5 a.u. Between these values of $d$, the second- and third-nearest neighbor exchange constants, $J_{2}$ and $J_{3}$, respectively, become of importance and $J_{2}$ even exceeds the value of $J_{1}$, resulting in a deep noncollinear ground state. Finally, at $d=5.5$ a.u. the nearest-neighbor exchange interaction is FM and in the long-range interaction the typical RKKY-like behavior is clearly visible. At this and larger distances $d$ the magnitude of the exchange constants, the depth of the spin-spiral ground state, and the energy difference between FM and AFM state becomes small due to a large separation between the atoms.

The transition from AFM-to-FM nearest-neighbor exchange coupling with increasing interatomic spacing is predicted from the Bethe-Slater curve (see, e.g., Ref. 47). The mechanism behind this effect can be understood within the Alexander-Anderson model ${ }^{33}$ of exchange interaction between the $d$ states of TM atoms as applied by Moriya. ${ }^{39}$ At small distances, the kinetic exchange interaction, which is AFM, dominates for a TM atom with nearly half-filled $d$-shell such as Mn. As the spacing is increased, the hopping $t$ and splitting between bonding and antibonding parts of the $d$-states decrease, while exchange splitting $\Delta$ normally increases. This leads to weakening of the kinetic exchange according to $t^{2} / \Delta$ dependence, outlined in the introduction. On the other hand, the FM double exchange, whose energy contribution is proportional to $t$, is also weakened upon stretching, but only linearly in the hopping. This leads to a crossover between negative $t^{2} / \Delta$ and positive $t$ parts of the exchange energy at a certain interatomic distance, and the chain becomes FM upon further stretching. We see in the next section how this competition is changed in favor of kinetic exchange due to hybridization with a substrate.

Within the so-called virtual crystal approximation (VCA) we artificially change the atomic number $Z$ of the atoms in the chain while keeping the charge neutrality and investigate the influence of the $3 d$-band filling on the noncollinear magnetism in Mn MWs. By doing this we aim at mimicking the behavior of the magnetic ground state upon alloying Mn with other elements, as well as charging upon, for example, deposition on a substrate. We perform these calculations at a fixed interatomic distance in the chain of $d=4.5$ a.u., roughly corresponding to the equilibrium interatomic distance of the TM chains in the middle of the $3 d$ series. $^{24}$ The atomic number $Z$ is varied between 24.0 and 26.0, corresponding to $\mathrm{Cr}$ and Fe chains, respectively, in steps of $\Delta Z=0.1-0.2$, which causes a shift of the Fermi level $E_{F}$ within the $3 d$ bands upward in energy with respect to the Fermi energy in the Cr chain.

The results of our calculations for the spin-spiral dispersion $E(q)$ are presented in Fig. 3(c) as a function of $Z$. We observe that, starting off with $\mathrm{Cr}(Z=24)$, which has a pronounced AFM ground state with an energy gain of more than $0.6 \mathrm{eV}$ with respect to the FM state, the energy difference between the FM state and the AFM state drops rapidly upon increasing the atomic number and vanishes at $Z \approx 25.3$. Upon further increasing $Z$, the FM state becomes favorable over the AFM configuration, and for an Fe chain with $Z=26$, the FM ground state is by more than $0.2 \mathrm{eV}$ lower in energy than the AFM solution, in accordance to previous collinear calculations at this distance. ${ }^{21}$ At values of $Z$ around 24.9 a small noncollinear ground state emerges close to the $X$ point, which shifts toward the $\Gamma$ point and becomes more pronounced in energy with increasing $Z$ until it disappears at $Z \approx 25.6$. The energy gain of the spin-spiral ground state with respect to the collinear solutions reaches as much as $75 \mathrm{meV}$ for $Z \approx 25.3$, compared to $38 \mathrm{meV}$ in a Mn MW with $Z=25$ [cf. Fig. 3(a)]. Overall, we observe a smooth transition between the dispersions of $\mathrm{Cr}$ and Fe chains.

From the spin-spiral dispersions we extract the Heisenberg exchange parameters for the first three neighbors, $J_{1}, J_{2}$, and $J_{3}$, as a function of $Z$, presented in Fig. 4(a), and analyze the trajectory that a monatomic chain takes in the magnetic phase space when its atomic number is changed between 24 and 26,
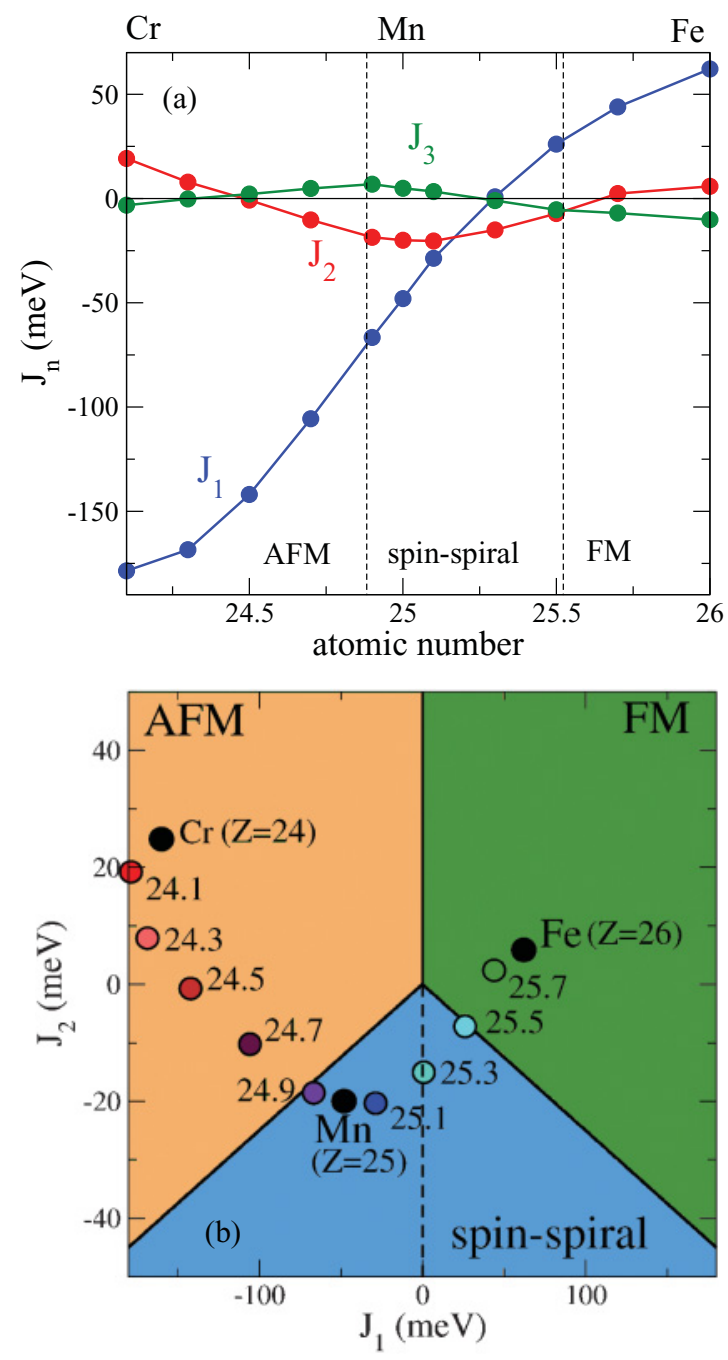

FIG. 4. (Color online) (a) Heisenberg exchange constants $J_{1}, J_{2}$, and $J_{3}$ as a function of the atomic number $Z$ for freestanding MWs with an interatomic distance of $d=4.5$ a.u. extracted from $E(q)$ given in Fig. 3(c). Dashed lines mark the boundaries of FM, AFM and spin-spiral ground states. (b) Phase diagram of the 1D Heisenberg model in the $\left(J_{1}, J_{2}\right)$-parameter space. The AFM phase is shaded in beige, the FM phase in green, and the spin-spiral phase is colored in blue. The circles represent the values of the Heisenberg exchange constants $J_{1}$ and $J_{2}$ given in (a). 
as shown in Fig. 4(b). From this plot we can see that mostly $J_{1}$ exceeds $J_{2}$ by an order of magnitude, while, in turn, $J_{3}$ is noticeably smaller than $J_{2}$ - thus, for our analysis we consider only $J_{1}$ and $J_{2}$ Heisenberg parameters and trace the position of our chains in the $J_{1}-J_{2}$ phase space. The magnetic ground state for the 1D Heisenberg model as a function of $J_{1}$ and $J_{2}$ is presented in Fig. 4(b).

We observe in Fig. 4(b) a smooth trajectory of the MW in the $J_{1}-J_{2}$ phase space. In the regime of $Z$ close to the pure $\mathrm{Cr}$ chain AFM nearest-neighbor exchange coupling prevails, that is, $J_{1}$ is about $-150 \mathrm{meV}$ and very large in comparison to $J_{2}$ with an absolute value of approximately $20 \mathrm{meV}$. Accordingly, in this region of $Z$ the ground state is AFM and the MWs are situated deeply inside the AFM region in the phase diagram of Fig. 4(b). When $Z$ approaches a value of $24.9, J_{1}$ drops significantly while $J_{2}$ is strengthened so that both exchange parameters are becoming closer in value. In the phase space, the chain therefore approaches the boundary between the AFM and spin-spiral phases. This boundary is crossed when $Z$ exceeds 24.9 and the chains reveal a noncollinear ground state until $Z$ reaches 25.5. In terms of exchange constants this happens when upon increasing atomic number, $J_{1}$ is dramatically decreased so that it becomes comparable to $J_{2}$. At $Z \approx 25.3$ $J_{1}$ vanishes; then it becomes positive and increases, exceeding $\left|J_{2}\right|$ for $Z>25.5$. Correspondingly, the chain becomes FM for $Z$ in the vicinity of Fe.

Remarkably, the dependence of $J_{1}$ on $Z$, displayed in Fig. 4(a), is very similar to that of the Bethe-Slater curve, which characterizes the sign of the exchange interaction as a function of $d / r_{d}$, with $d$ being the distance between the TM atoms and $r_{d}$ as the spread of the $d$ orbitals. It was demonstrated from $a b$ initio calculations in Ref. 21 that the $3 d$ TM monatomic chains display the Bethe-Slater behavior as a function of $d$, with the chain's position on this curve corresponding to $r_{d}$ (cf. Fig. 3 in Ref. 21). The Bethe-Slater-like behavior of $J_{1}(Z)$ in Fig. 4(a) is another evidence of the applicability of this simple picture for $3 d$ TM chains: At fixed interatomic distance $d$ (of 4.5 a.u.) upon varying the nuclear charge $Z$ the spread of $d$ orbitals is changed. For smaller $Z$ the $r_{d}$ is larger and for larger $Z$ the spread is smaller; ${ }^{48}$ this results in positioning of the AFM Cr and FM Fe chains on different sides of the BetheSlater curve, with Mn chains in the middle at the FM-AFM crossover.

A different point of view can be taken by referring to the model of Moriya, ${ }^{39}$ which is an extension of the Alexander and Anderson model ${ }^{33}$ for the exchange interaction between TMs as a function of the number of electrons in the $d$ shell. Moriya finds a curve similar to that of Bethe-Slater if he varies the number of $3 d$ electrons of the interacting TM atoms, in complete analogy to $J_{1}(Z)$ presented in Fig. 4(a). This confirms that while the kinetic exchange mechanism is behind the AFM ordering of TMs with half-filled $d$ shell, the double exchange leads to FM ground state for TMs with half-filled $d$-spin-subband. The link between the Bethe-Slater and Moriya pictures of exchange, as outlined in the previous paragraph, is the decrease of the spread of the $d$ orbitals, $r_{d}$, upon increasing electronic occupation in TM series. ${ }^{48}$ Effectively, as far as Mn chains are concerned, we can therefore conclude that the effect of the band filling on the magnetic state is the same as that from changing the interatomic distance $d$ in the chain.
For the next-nearest and next-next-nearest neighbor exchange interactions, $J_{2}$ and $J_{3}$, we find two changes of sign in the considered range of atomic numbers [cf. Fig. 4(a)]. This result is in nice qualitative agreement with the spin susceptibilities for bulk Fe calculated by Terakura et al. ${ }^{49}$ as a function of the energy. As the increase of the atomic number in our approach leads to a filling of the $3 d$ band we can compare our result with the spin susceptibilities.

\section{NONCOLLINEAR MAGNETISM IN DEPOSITED Mn CHAINS}

For supported Mn chains we consider three different metallic surfaces: unreconstructed $\mathrm{Cu}(110), \mathrm{Ag}(110)$, and $\operatorname{Pd}(110)$. This choice is motivated by several reasons. First, these substrates are rather promising experimentally as they provide trenches for chain self-assembly, while chain creation atom by atom by manipulation with an STM tip may also be feasible. Second, as far as we assume that the chains grow pseudomorphically on these surfaces, the interatomic distance between the chain atoms $d$ is dictated by the lattice constant of the substrate, taking values of 4.82, 5.30, and 5.59 a.u. for $\mathrm{Cu}(110), \operatorname{Pd}(110)$, and $\mathrm{Ag}(110)$, respectively. In the previous section we observed that in this regime of interatomic distance Mn chains display a noncollinear ground state, while among the collinear solutions freestanding Mn chains favor an AFM state for $d$ corresponding to that of $\mathrm{Cu}(110)$, while for the spacing corresponding to $\operatorname{Pd}(110)$ and $\operatorname{Ag}(110)$ the chains prefer a FM configuration. Finally, while $\mathrm{Cu}$ and $\mathrm{Ag}$ are noble metals with predominantly $s$ character of electronic states at the Fermi level, the degree of hybridization of Mn atoms and the underlying $\mathrm{Cu}$ or $\mathrm{Ag}$ substrate is much weaker than that of Mn chains deposited on Pd, which has high density of states of $4 d$ electrons at the Fermi energy. We can thus compare the effect of hybridization on the exchange interaction of deposited Mn MWs.

The summary of our calculations is presented in Fig. 5, where the energy dispersion curves $E(q)$ are plotted for $\mathrm{Mn}$

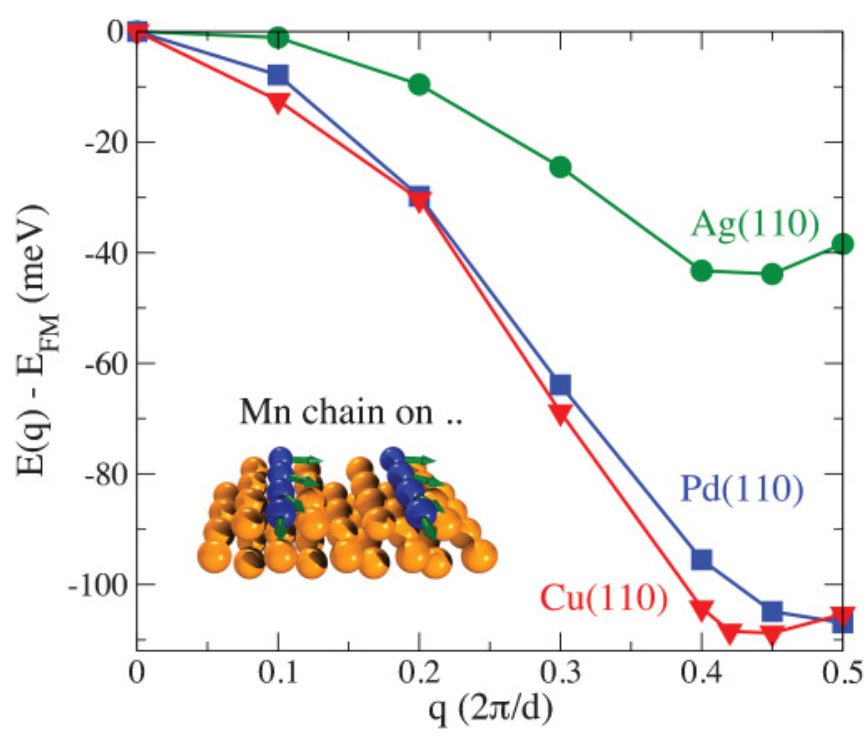

FIG. 5. (Color online) Self-consistently calculated flat spin-spiral dispersion curves $E(q)$ for a $\mathrm{Mn}$ chain on $\mathrm{Cu}(110)$ (red triangles), $\operatorname{Ag}(110)$ (green circles), and $\operatorname{Pd}(110)$ (blue squares). 
chains on $\mathrm{Cu}(110), \operatorname{Pd}(110)$, and $\mathrm{Ag}(110)$ as a function of the $q$ vector of a flat spin-spiral propagating along the chain. We observe that in all cases the energetically favorable state is AFM among the collinear solutions, even for Mn chains on $\operatorname{Pd}(110)$ and $\mathrm{Ag}(110)$, which prefer FM ordering in an unsupported situation. This tendency toward antiferromagnetism in surface-deposited chains and small clusters of $3 d$ TMs has been observed previously in Refs. 18 and 21 and stems from the hybridization between the chain and the substrate. As far as the noncollinear solutions are concerned, we predict that despite strong modifications in the shape of $E(q)$ and a tendency toward AFM ordering, a shallow spin-spiral energy minimum remains for $\mathrm{Mn}$ chain on $\mathrm{Cu}(110)$ (3.5 meV below the AFM state) and on $\mathrm{Ag}(110)$ (5.5 meV below the AFM solution). In contrast, the strong hybridization between the $4 d$ states of the Pd surface and the $3 d$ states of $\mathrm{Mn}$ atoms around the Fermi energy leads to a disappearance of the spin-spiral minimum, and the resulting order is AFM.

In order to investigate the influence of the substrate on exchange interactions of deposited Mn chains in more detail, we choose the most striking example of a Mn MW on $\mathrm{Ag}(110)$. In Fig. 6(a) we present the self-consistently calculated flat spin-spiral dispersion curve for a Mn chain on $\operatorname{Ag}(110)$ in comparison to the dispersion curve of a freestanding Mn chain at the same interatomic distance of $d=5.59$ a.u. We observe
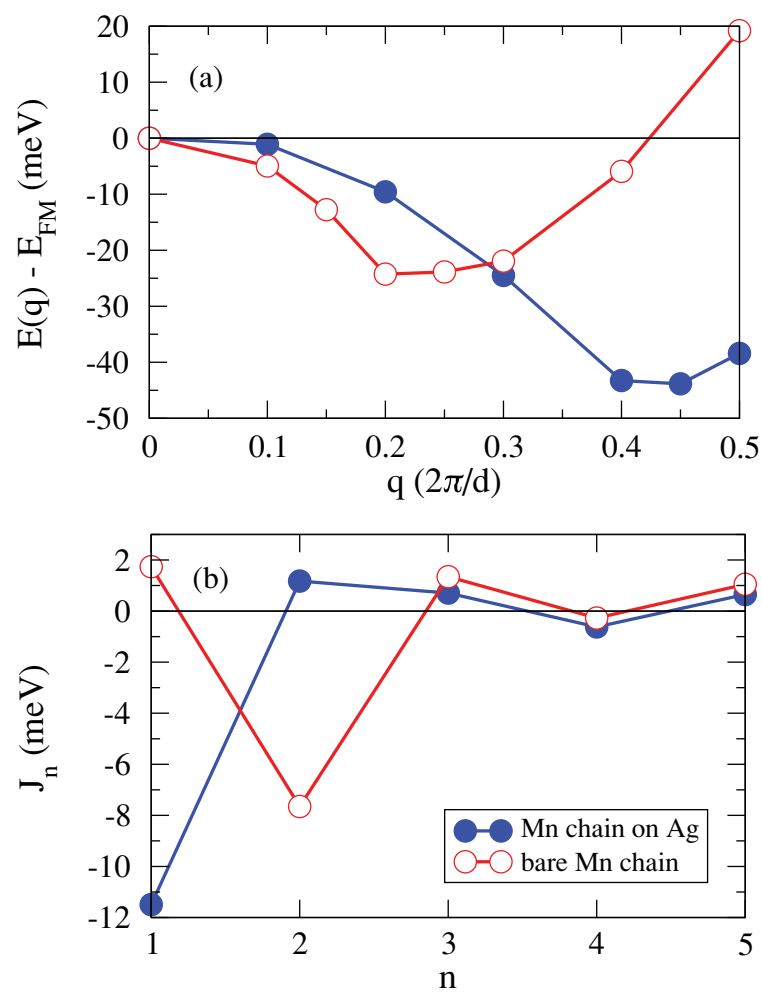

FIG. 6. (Color online) (a) Flat spin-spiral energy dispersion calculated self-consistently for a Mn chain on $\operatorname{Ag}(110)$ (solid blue circles) and a freestanding Mn MW with the same interatomic spacing of $d=5.59$ a.u. (open red circles). (b) Heisenberg exchange constants extracted from spin-spiral dispersion curves in (a). Solid blue circles show the $J_{n}$ for a Mn chain on a $\operatorname{Ag}(110)$ surface and open red circles represent the Heisenberg constants for a freestanding Mn wire with the same interatomic distance of $d=5.59$ a.u. that while the freestanding Mn MW exhibits a significant energy minimum of $\Delta E=25 \mathrm{meV}$ with respect to the FM solution of a spin-spiral with an angle of $\alpha_{\min }=72^{\circ}$ between neighboring spins, the spin-spiral state becomes much less favorable for the chain on the $\operatorname{Ag}(110)$ substrate $(\approx 5.5 \mathrm{meV}$ below the AFM state) and the angle shifts to a larger value of $\alpha_{\min }=151^{\circ}$. As already pointed out, the energy difference between the FM and the AFM solution is reversed for a Mn chain deposited on the substrate. While the FM state is by 20 meV lower than the AFM state for the freestanding Mn chain, the AFM state becomes by $40 \mathrm{meV}$ more favorable for the deposited Mn chain. The comparison of the exchange parameters, Fig. 6(b), which were extracted from the dispersion curves in Fig. 6(a), underlines the influence of the substrate on the nature of exchange interactions in this system: While the exchange constants beyond the second nearest-neighbor are similar for the freestanding and Ag-supported Mn chain, the $\operatorname{Ag}(110)$ surface causes a change in sign of the parameters $J_{1}$ and $J_{2}$, as compared to the freestanding Mn MW. Further, the magnitude of $J_{1}$ increases significantly, emphasizing predominantly AFM coupling along the deposited chain.

It seems fruitful to analyze the correspondence between the changes in the local Mn spin moment $\mu_{S}^{\mathrm{Mn}}$ upon deposition and the change in the nature of exchange coupling. In general, as was already reported in Ref. 21 for the FM and the AFM states of $\mathrm{Mn}$ chains on the (110) surfaces of $\mathrm{Cu}, \mathrm{Ag}$, and $\mathrm{Pd}$, the value of $\mu_{S}^{\mathrm{Mn}}$ (in units of $\mu_{B}$ ) is reduced in the FM (AFM) state from 3.97 (3.98) for an unsupported Mn chain to 3.69 (3.75) on $\mathrm{Cu}(110)$, from 4.12 (4.20) for an unsupported Mn chain to 4.00 (3.96) on $\operatorname{Pd}(110)$, and from 4.20 (4.29) for an unsupported Mn chain to 4.05 (4.12) on $\operatorname{Ag}(110)$. This reduction of the spin moment comes from spin-dependent changes in the number of electrons inside the Mn atomic spheres. While the changes in the local Mn spin moment stemming from $s$ electrons are rather small upon deposition, owing to small spin polarization of these states, the changes in the $d$ moment and $d$ occupation for up and down spins are rather significant. For example, in the case of the AFM Mn chain the number of $d$ electrons in the spin-up channel is changed from 4.47 in the freestanding state to 4.39 upon deposition on $\operatorname{Ag}(110)$, while the corresponding values for the spin-down channel constitute 0.27 and 0.37 , respectively.

The values for the changes in the Mn $d$ occupation upon deposition are quite similar for other substrates and magnetic states of deposited Mn chains and a general trend emerges: Upon deposition we observe a decrease in the occupation of spin-up electrons, while the number of spin-down electrons increases, with consequent reduction of the total Mn spin moment. In a simple picture of the densities of states (DOS) of a freestanding chain, this situation corresponds to an effective decrease in the value of the interatomic distance $d$, when the chain is deposited on a surface: The spin-down $d$ states (mostly unoccupied in $\mathrm{Mn}$ ) are shifted to lower energies while the spin-up $d$ states (mostly occupied in Mn) are shifted in the opposite direction, and the overall exchange splitting and the value of the spin moment is reduced. Qualitatively, as we discovered in the previous section, this reduction in interatomic distance has the same effect as the alloying of the $\mathrm{Mn}$ wire with $\mathrm{Cr}$ atoms, which results in large and negative $J_{1}$ exchange parameter, see Figs. 3(b) and 4(b), and the 


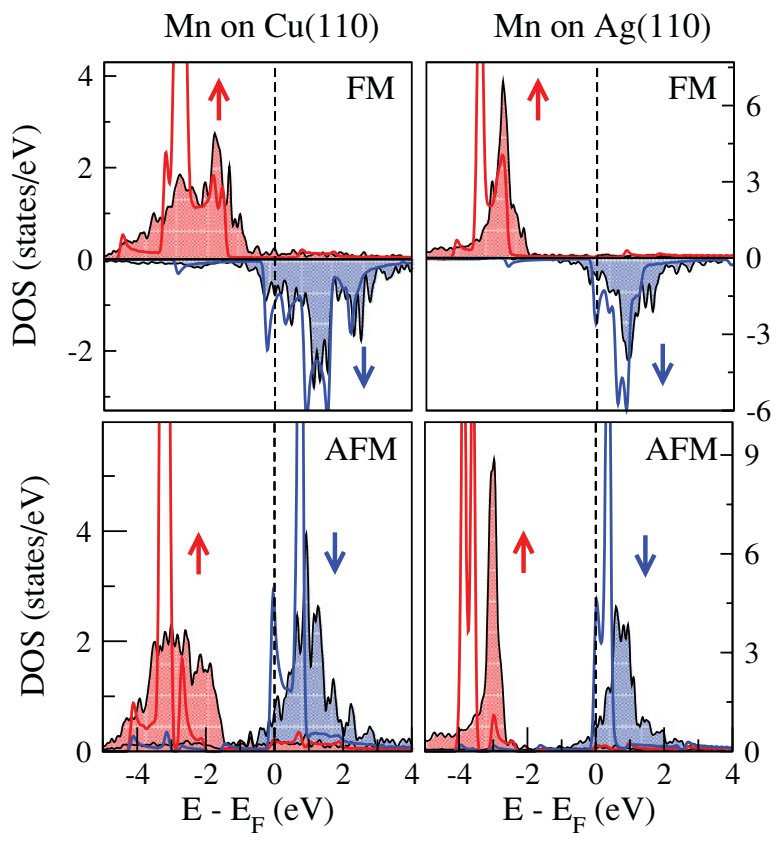

FIG. 7. (Color online) Local density of states (DOS) of a Mn atom in a chain deposited on $\mathrm{Cu}(110)$ (left panels) and $\mathrm{Ag}(110)$ (right panels) in comparison to the Mn DOS of a bare chain at the corresponding lattice constant, both for ferromagnetic (FM) and antiferromagnetic (AFM) arrangement of the spins. While the DOS of the freestanding chains is given by a solid line, the DOS of the deposited Mn chains corresponds to the shaded areas. Red (blue) lines, red (blue) shading, and red (blue) arrows mark the spin-up (spin-down) DOS.

exchange interaction in deposited Mn chains leans toward strong antiferromagnetism, as observed in Fig. 6.

The reduction of exchange splitting $\Delta$ of the Mn $d$ states upon deposition on a substrate can be seen also from the local DOS of deposited chains in comparison to the DOS in the freestanding configuration. In Fig. 7 we compare the DOS of bare and $\mathrm{Mn}$ chains deposited on $\mathrm{Cu}(110)$ and $\mathrm{Ag}(110)$ substrates for both collinear spin configurations, that is, FM and AFM states. The reduction of $\Delta$, defined as the difference between the center of mass of spin-up (red) and spin-down (blue) Mn states, upon deposition on the surface can be easily seen in these plots. It is especially pronounced for the case of a Mn chain deposited on $\operatorname{Ag}(110)$, in both the FM and the AFM configuration. In this case the distance $d$ between the Mn atoms along the chain is larger and the corresponding peaks in the DOS are sharper than in the case of the Mn chain deposited on $\mathrm{Cu}(110)$ which imposes a smaller Mn-Mn distance. As can be seen, upon deposition on $\operatorname{Ag}(110)$ the value of the exchange splitting can be reduced by as much as $0.3-0.4 \mathrm{eV}$ as compared to the freestanding value of $\Delta$. In case of $\mathrm{Cu}(110)$ the $\mathrm{Mn}$ states are rather spread in energy for both structural cases and the center of mass of the $d$ bands is more difficult to determine, however, even in this case the reduction of the exchange energy upon deposition is quite visible.

In terms of the Alexander-Anderson model, outlined in the previous sections, the reduction in the value of $\Delta$ promotes the AFM kinetic exchange over FM double exchange between the $\mathrm{Mn}$ atoms. Another subtle point in this competition, which was pointed out in Ref. 18, is that the gain in energy due to double exchange is smaller for a chain on a surface than it is for a free-standing chain. If the $d$ states are resonant with the background hybridization, for example, due to the presence of a substrate, the tails of the bonding and antibonding resonances cross the Fermi energy, and their repopulation partly counteracts the energy gain due to double exchange in a bare chain. ${ }^{18}$ At the end, due to enhancement of the kinetic exchange and weakening of the double exchange, Mn chains, situated right at the FM-AFM crossover point, become AFM when deposited on a substrate.

\section{SIMULATION OF SPIN-POLARIZED STM IMAGES OF NONCOLLINEAR Mn CHAINS}

An experimental verification of the predicted noncollinear magnetic ground state of $\mathrm{Mn}$ chains on $\operatorname{Ag}(110)$ and on $\mathrm{Cu}(110)$ is very challenging as the atomic chain structure needs to be revealed and individual chains have to be addressed. In addition, the magnetic signal is small due to the low amount of magnetic material and in any spin-spiral state the magnetization is compensated on the atomic length scale. Therefore, there are few experimental techniques suitable for this challenge. In recent years, it has been demonstrated that spin-polarized scanning tunneling microscopy (SP-STM) is a powerful tool to image magnetic order of individual nanostructures down to the atomic scale ${ }^{4,50-52}$ and noncollinear spin structures in ultrathin films have been observed. ${ }^{35,36,53,54}$ As calculated SP-STM images based on the spin-polarized extension of the Tersoff-Hamann model ${ }^{55}$ are in excellent agreement with the experimental data, we have performed such simulations for the system of deposited atomic chains, taking $\mathrm{Mn}$ chains on $\mathrm{Cu}(110)$ as an example.

Before we turn to the predicted SP-STM images, it is insightful to analyze the partial charge and magnetization density obtained from our first-principles calculations in the vicinity of the Fermi energy. As we are interested in the contrast obtainable with SP-STM, we depict these quantities in the vacuum a few Ångströms above the Mn chain atoms. Figure 8 displays such a top view of the $\mathrm{Mn}$ chain on $\mathrm{Cu}(110)$ with a spin-spiral magnetic state characterized by the vector $q=0.4 \times \frac{2 \pi}{a}$. This magnetic structure is close to the absolute minimum in total energy (see Fig. 5), and it is commensurate with the atomic lattice with a relatively small pitch of five atoms allowing a simple discussion.

In the magnetization density (top panel of Fig. 8) the location of the Mn atoms can be clearly seen while the vacuum charge density (bottom panel) only resolves the chain direction. The $x$ and $y$ direction of the magnetization (center panels) already hint at the contrast expected with a magnetic STM tip which is sensitive to a magnetization component given by its own magnetization direction. We find that the maxima and minima need not correspond to the atom positions and that the images can change qualitatively with the tip magnetization. A further striking point is evident from the local magnetization (top panel); that is, the rotation of the magnetization density is not uniform. Above the atom sites the rotation is slow and in between it is much faster.

Another interesting feature which can be seen in the upper plot of Fig. 8 is that the direction of the magnetization in the 

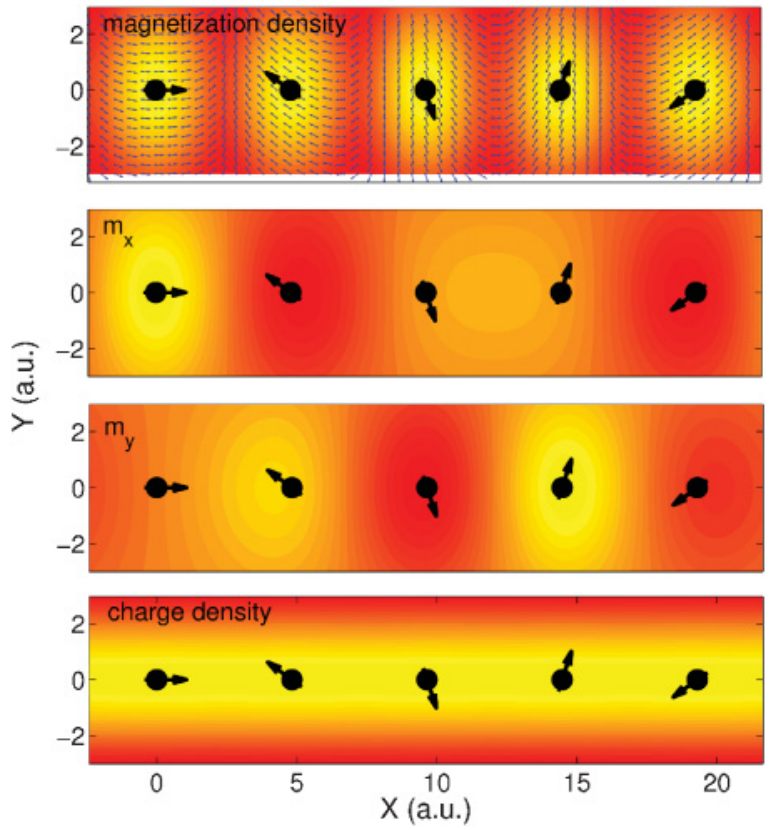

FIG. 8. (Color online) Local vacuum charge and magnetization density of a $\mathrm{Mn}$ chain on $\mathrm{Cu}(110)$ in a spin-spiral state with $q=$ $0.4 \times \frac{2 \pi}{a}$ at a height of $z=5.9 \AA$ above the chain and for an energy interval of $E=\left[E_{F}-300 \mathrm{meV}, E_{F}\right]$. The chain extends along the $x$ direction and the black circles represent the position of the Mn atoms with arrows indicating their magnetic moments. The top panel shows the magnetization density: whereas the background color denotes the absolute value of the local magnetization [yellow (red) corresponding to high (low) values], the small blue arrows indicate the unit vector of its direction. The second and the third panels display the $x$ and $y$ components of the magnetization, respectively, while the bottom panel shows the vacuum charge density.

vacuum coincides with that inside the Mn atoms. This appears somewhat counterintuitive, since, according to the density of states of the Mn chain on $\mathrm{Cu}(110)$ presented in Fig. 7, the minority states dominate in the region around the Fermi level. In order to resolve this discrepancy, in Fig. 9 we plot the spin- and orbitally resolved local DOS inside the Mn spheres together with the LDOS in the vacuum at a distance of $z=5.9 \AA$ from the Mn chain on $\mathrm{Cu}(110)$ in its FM state (the case of the AFM Mn chain is essentially analogous). The orbitally resolved LDOS [Fig. 9(a)] shows that these are the Mn $d$ states which dominate at $E_{F}$ for both spin directions, and while for spin-down the $d$-DOS is quite large, for spin-up it is rather small and featureless, owing to the exchange splitting which shifts the position of the spin-up $d$ states deep below the Fermi level. On the other hand, the situation with the LDOS in the vacuum is completely opposite, as can be seen from Fig. 9(b). For minority spin, the vacuum LDOS around $E_{F}$ is very small, increasing drastically in value only above $1 \mathrm{eV}$. However, the vacuum LDOS of spin-up electrons is very large and dominates the total LDOS in a large window around $E_{F}$ and determines the direction of the local magnetization DOS in the vacuum above the $\mathrm{Mn}$ atoms, presented in Fig. 8.

By comparing Figs. 9(a) and 9(b) we can see that the structure of the spin-up vacuum LDOS in the energy window $[-2 \mathrm{eV},+1 \mathrm{eV}]$ is in very nice correspondence to the $s$-DOS of

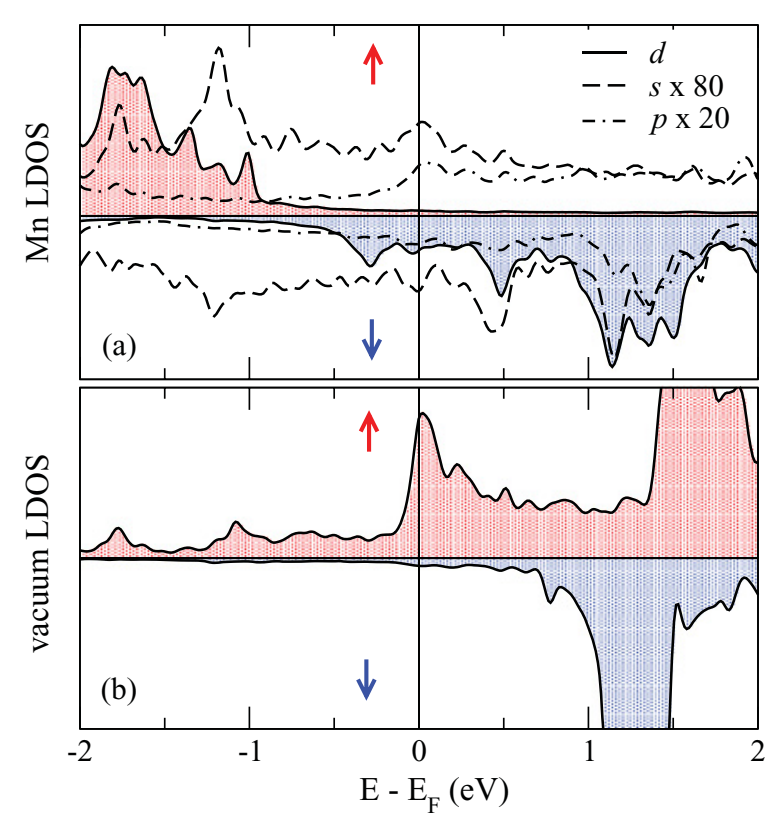

FIG. 9. (Color online) (a) Local density of states of a Mn atom in a FM chain deposited on $\mathrm{Cu}(110)$ decomposed into $s, p$, and $d$ contributions. The $s$ - and $p$-DOS are multiplied by a factor of 80 and 20, respectively. (b) Corresponding local DOS in the vacuum at a height of $z=5.9 \AA$ above the chain. In both plots, red (blue) shading and red (blue) arrows mark the spin-up (spin-down) DOS. The units are arbitrary.

the $\mathrm{Mn}$ atom. In particular, the peaks in the $s$-DOS at around $-1.8,-1.1,+0.25$, and $+0.5 \mathrm{eV}$ and the peak directly at the Fermi energy can be clearly seen also in the majority vacuum LDOS, while the $d$-DOS is quite featureless for energies above $-1 \mathrm{eV}$ in this spin channel. This suggests that the magnetization in the vacuum high above the chain is actually driven by spin-polarized $s$ and $p$ electrons which reach further into the vacuum, rather than localized $d$ states. The spin polarization of the $s$ and $p$ states stems from their hybridization with the $d$ electrons in the chain, which influence their bonding properties. While the majority $s$ and $p$ states bare an antibonding character with respect to the interaction with the substrate, the minority $s$ and $p$ states are bonded stronger to the $\mathrm{Cu}(110)$ surface, which makes their spread into the vacuum much smaller. The latter scenario has been also recently observed and explained from first principles for deposited magnetic adatoms and their clusters ${ }^{4,56}$ as originating from the reduced coordination and symmetry of the adsorbates. This explains why the localized Mn spin moment due to spin-up states well below the Fermi energy is collinear with the magnetization density in the vacuum, obtained by integrating the states in a small window around $E_{F}$. Such a correspondence between the direction of the atomic spin moment in the deposited chain and the vacuum magnetization was also found for biatomic Fe chains on an Ir substrate. ${ }^{10}$

Now we turn to the calculated spin-polarized STM images which have been obtained applying the spin-polarized version of the Tersoff-Hamann model ${ }^{55}$ and using the electronic structure calculated within DFT. Figure 10 displays the result for a tip magnetization along the $y$ direction. The upper two panels show the non-spin-polarized and the spin-polarized 


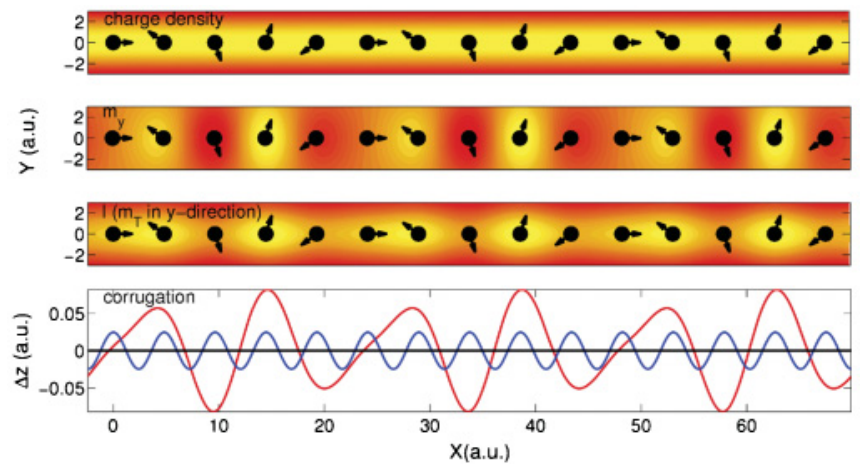

FIG. 10. (Color online) Calculated SP-STM images for a Mn chain on $\mathrm{Cu}(110)$ in a spin-spiral state with $q=0.4 \times \frac{2 \pi}{a}$. A height of $z=5.9 \AA$, a bias voltage of $-0.3 \mathrm{~V}$, and a tip spin-polarization of 0.4 have been chosen. The tip magnetization direction is assumed along the $y$ direction. In the upper three panels the black spheres show the Mn atoms and the arrows indicate their spin moments which show the magnetic periodicity of five atoms. The upper two panels display the non-spin-polarized and the spin-polarized component of the tunneling current, respectively. The third panel depicts the resulting total tunneling current. The bottom panel shows line scans along the chain axis obtained by simulating the constant current mode. The red line corresponds to the corrugation in the spin-polarized case. The corrugation for the non-spin-polarized case (blue line) is given as a reference for the atom positions and has been enhanced by a factor of 25 .

component of the tunneling current, respectively, at a fixed distance from the chain atoms of $5.9 \AA$. As expected, the atom positions are not resolved in the non-spin-polarized part of the current within the Tersoff-Hamann approximation. The apparent width of the chain is about $2 \AA$. The spin-polarized part, on the other hand, clearly shows the five-atom periodicity of the spin spiral structure with $q=0.4 \times \frac{2 \pi}{a}$, which implies an angle of $\alpha=144^{\circ}$ between the spins of neighboring atoms. In the total tunneling current, the superstructure due to the magnetic signal is still visible, but it is slightly damped due to the large contribution from the non-spin-polarized part. Therefore, the spin structure should be resolvable within an SP-STM experiment.

In order to quantify the contrast we have calculated the corrugation amplitude by simulating a line scan along the chain axis in the constant-current mode. We have chosen the starting point of the scan lines at a height of $5.9 \AA$, which fixes the constant current. The obtained scan lines, shown in the bottom panel of Fig. 10, reveal an asymmetric profile and the maxima and minima of the spin-polarized STM image do not necessarily coincide with atom positions. We find a corrugation amplitude, that is, the maximum height change as the tip moves along the chain, of about $0.08 \AA$. This value is above the resolution limit of STM (cf. measurements of spin spirals in Ref. 35) and is enhanced at smaller tip-sample distance and larger tip spin-polarization (a value of 0.4 has been assumed here).

In Fig. 11 the predicted SP-STM images are displayed for a tip magnetization along the $x$ axis. The five-atom periodicity is again apparent from the plot and a threefold contrast pattern is also observed. The main difference between a tip magnetization, $\mathbf{m}_{T}$, pointing into the $x$ and $y$ direction,

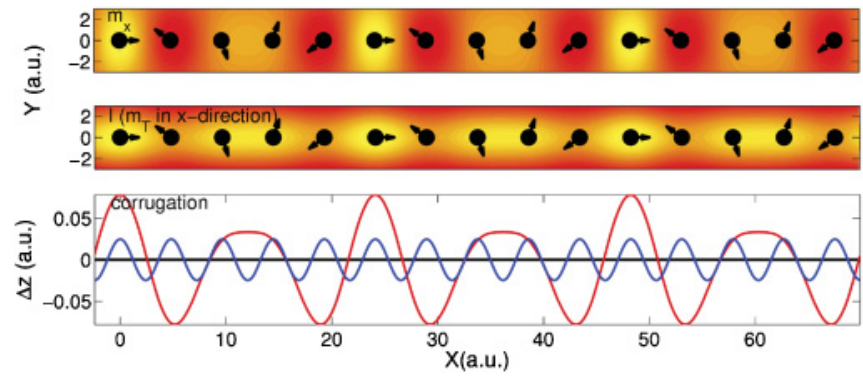

FIG. 11. (Color online) Analogous to Fig. 10, but with the tip magnetization direction pointing along the $x$ direction.

respectively, is that there are atoms whose magnetic moments point directly into the $x$ direction, but there are no atoms whose magnetic moments directly point into the $y$ direction. At some points the maximum of the spin-polarized corrugation is therefore directly localized above the atom sites in the case of $\mathbf{m}_{T}$ pointing in the $x$ direction, while when $\mathbf{m}_{T}$ pointing in the $y$ direction this is never the case. For this reason, the two schemes are not only shifted with respect to each other, but look substantially different. In Fig. 11 the third and the fourth atoms are equivalent in the sense that their magnetizations have the same $\alpha$ component and therefore they produce the same contrast. The same argument holds for atoms 2 and 5 . On the contrary, in the case of $\mathbf{m}_{T}$ pointing in the $y$ direction these atoms are not equivalent.

Interestingly, the vacuum magnetization can be well reproduced within a simple model of SP-STM, ${ }^{57}$ which relies only on a knowledge of the magnetic structure and assumes a spherical exponential decay of the wave functions. Therefore, the discussed SP-STM images could have also been obtained within this much faster approach. Simulations for spin spirals with a different pitch, that is, other values of $q$, are therefore easily possible in this way.

\section{SUMMARY AND OUTLOOK}

We have performed an ab initio study of the noncollinear magnetism in freestanding and deposited monatomic Mn chains neglecting the effect of spin-orbit coupling, which is relatively small in our systems. We find that the spin-spiral magnetic state is the ground state of unsupported Mn chains in a large interval of distance between the atoms. We were able to attribute the appearance of noncollinear magnetism in this system to the existence of a FM-to-AFM transition point. In the vicinity of this point the nearest-neighbor exchange parameter changes sign and is on the order of exchange interactions beyond nearest neighbors, which promotes an energy minimum of a spin-spiral state. We also demonstrate, within the VCA, that upon alloying chains of $\mathrm{Mn}$ with $\mathrm{Cr}$ and $\mathrm{Fe}$ the ground state of $\mathrm{Mn}$ chains can be controlled. In particular, while Cr-rich Mn chains are antiferromagnets, upon increasing the Mn concentration they exhibit a spin spiral ground state. Upon mixing Mn chains with Fe a transition from a noncollinear ground state to the FM state occurs. The trajectory which Mn chains take in the magnetic phase space upon alloying with $\mathrm{Cr}$ and $\mathrm{Fe}$ is therefore smooth. 
For Mn chains on the (110) surfaces of fcc $\mathrm{Cu}, \mathrm{Pd}$, and Ag we observe an overall strong tendency toward antiferromagnetism. As a result, among the considered substrates only $\mathrm{Cu}(110)$ and $\mathrm{Ag}(110)$ allow for a spin-spiral ground state in the deposited Mn chains close to the AFM solution with a modest gain in energy of 3.5 and $5.5 \mathrm{meV}$ per Mn atom over the AFM state, respectively. We attribute this suppression of the tendency of Mn chains toward noncollinear magnetism, which is extremely pronounced for unsupported chains, to the effect of the hybridization with the substrate. Within the Alexander-Anderson model of exchange between $d$ states it can be shown that the hybridization weakens the FM double exchange mechanism and the AFM kinetic exchange interaction prevails. ${ }^{18}$

An effect which can enhance the spin-spiral minimum of Mn chains on heavier substrates lies in the spin-orbit driven Dzyaloshinskii-Moriya interaction (DMI). ${ }^{35}$ Irrespective of its sign, adding the DMI contribution to the spin-spiral dispersion curve would make the spin-spiral minimum in the vicinity of the collinear AFM solution more pronounced, owing to the antisymmetric nature of this energy correction with respect to the spin-spiral vector. The DMI energy contribution will have to compete with another manifestation of the spin-orbit coupling in these systems - the magnetocrystalline anisotropy energy, which we, however, expect to be rather small for considered deposited chains.

Taking into account the stabilization of the magnetization by the weak spin-orbit coupling in $\mathrm{Mn}$ chains on the $\mathrm{Cu}$ and Ag substrates, we predict that the spin-spiral state in deposited Mn chains can be observed experimentally via, for example, SP-STM techniques. Based on the vacuum charge and magnetization density of supported $\mathrm{Mn}$ chains on $\mathrm{Cu}(110)$ we calculate SP-STM images, which can provide a conclusive evidence for a noncollinear ground-state spin structure in $\mathrm{Mn}$ chains.

\section{ACKNOWLEDGMENTS}

Financial support of the Stifterverband für die Deutsche Wissenschaft is gratefully acknowledged. Y.M. thanks HGFYIG Programme VH-NG-513 for funding and Jülich Supercomputing Center for computational time. We would like to thank Gustav Bihlmayer and especially Phivos Mavropoulos and Stefan Blügel for many illuminating discussions and suggestions.
*Permanent address: Fritz-Haber-Institute der Max-PlanckGesellschaft, Berlin, Germany.

†y.mokrousov@fz-juelich.de

${ }^{1}$ P. Gambardella, A. Dallmeyer, K. Maiti, M. C. Malagoli, W. Eberhardt, K. Kern, and C. Carbone, Nature (London) 416, 301 (2002).

${ }^{2}$ C. F. Hirjibehedin, C. P. Lutz, and A. J. Heinrich, Science 312, 1021 (2006).

${ }^{3}$ M. R. Calvo, J. Fernández-Rossier, J. J. Palacios, D. Jacob, D. Natelson, and C. Untied, Nature (London) 458, 1150 (2009).

${ }^{4}$ D. Serrate, P. Ferriani, Y. Yoshida, S.-W. Hla, M. Menzel, K. von Bergmann, S. Heinze, A. Kubetzka, and R. Wiesendanger, Nat. Nanotech. 5, 350 (2010).

${ }^{5}$ A. Smogunov, A. D. Corso, and E. Tosatti, Nat. Nanotech. 3, 22 (2008).

${ }^{6}$ A. Delin, E. Tosatti, and R. Weht, Phys. Rev. Lett. 92, 057201 (2004).

${ }^{7}$ Y. Mokrousov, G. Bihlmayer, S. Heinze, and S. Blügel, Phys. Rev. Lett. 96, 147201 (2006).

${ }^{8}$ A. Sokolov, C. Zhang, E. Y. Tsymbal, and J. Redepenning, Nat. Nanotech. 2, 171 (2007).

${ }^{9}$ S. Lounis, P. Dederichs, and S. Blügel, Phys. Rev. Lett. 101, 107204 (2008).

${ }^{10}$ Y. Mokrousov, A. Thiess, and S. Heinze, Phys. Rev. B 80, 195420 (2009).

${ }^{11}$ P. Lucignano, R. Mazzarello, A. Smogunov, M. Fabrizio, and E. Tosatti, Nat. Mater. 8, 563 (2009).

${ }^{12}$ O. A. Tretiakov and A. Abanov, Phys. Rev. Lett. 105, 157201 (2010).

${ }^{13}$ D. S. G. Bauer, P. Mavropoulos, S. Lounis, and S. Blügel, e-print arXiv:1010.4730 (to be published).
${ }^{14}$ R. H. M. Smit, C. Untiedt, A. I. Yanson, and J. M. van Ruitenbeek, Phys. Rev. Lett. 87, 266102 (2001).

${ }^{15}$ L. Hammer, W. Meier, A. Schmidt, and K. Heinz, Phys. Rev. B 67, 125422 (2003).

${ }^{16}$ P. Segovia, D. Purdie, M. Hengsberger, and Y. Baer, Nature (London) 402, 504 (1999).

${ }^{17}$ S. Lounis, P. Mavropoulos, R. Zeller, P. H. Dederichs, and S. Blügel, Phys. Rev. B 75, 174436 (2007).

${ }^{18}$ P. Mavropoulos, S. Lounis, and S. Blügel, Phys. Status Solidi B 247, 1187 (2010).

${ }^{19}$ P. Ruiz-Diaz, J. L. Ricardo Chavez, J. Dorantes-Davila, and G. M. Pastor, Phys. Rev. B 81, 224431 (2010).

${ }^{20}$ J. Mejia-Lopez, A. H. Romero, M. E. Garcia, and J. L. MoranLopez, Phys. Rev. B 78, 134405 (2008).

${ }^{21}$ Y. Mokrousov, G. Bihlmayer, S. Blügel, and S. Heinze, Phys. Rev. B 75, 104413 (2007).

${ }^{22}$ R. Mazzarello and E. Tosatti, Phys. Rev. B 79, 134402 (2009).

${ }^{23}$ H. Hashemi, W. Hergert, and V. S. Stepanyuk, Phys. Rev. B 81, 104418 (2010)

${ }^{24}$ J. C. Tung and G. Y. Guo, Phys. Rev. B 76, 094413 (2007).

${ }^{25}$ J. C. Tung and G. Y. Guo, Phys. Rev. B 81, 094422 (2010).

${ }^{26}$ M. Urdaniz, M. Barral, and A. Llois, Physica B 404, 2822 (2009).

${ }^{27}$ M. Zelený, M. Šob, and J. Hafner, Phys. Rev. B 80, 144414 (2009).

${ }^{28}$ H. Hashemi, G. Fischer, W. Hergert, and V. Stepanyuk, J. Appl. Phys. 107, 09E311 (2010).

${ }^{29}$ C. Ataca, S. Cahangirov, E. Durgun, Y.-R. Jang, and S. Ciraci, Phys. Rev. B 77, 214413 (2008).

${ }^{30}$ H. J. Lee, W. Ho, and M. Persson, Phys. Rev. Lett. 92, 186802 (2004).

${ }^{31}$ F. Meier, L. Zhou, J. Wiebe, and R. Wiesendanger, Science 320, 82 (2008). 
${ }^{32}$ L. Zhou, J. Wiebe, S. Lounis, E. Vedmedenko, F. Meier, S. Blügel, P. Dederichs, and R. Wiesendanger, Nat. Phys. 6, 187 (2010).

${ }^{33}$ S. Alexander and P. W. Anderson, Phys. Rev. 133, A1594 (1964).

${ }^{34}$ P. Ferriani, I. Turek, S. Heinze, G. Bihlmayer, and S. Blügel, Phys. Rev. Lett. 99, 187203 (2007).

${ }^{35}$ M. Bode, M. Heide, K. von Bergmann, P. Ferriani, S. Heinze, G. Bihlmayer, A. Kubetzka, O. Pietzsch, S. Blügel, and R. Wiesendanger, Nature (London) 447, 190 (2007).

${ }^{36} \mathrm{P}$. Ferriani, K. von Bergmann, E. Y. Vedmedenko, S. Heinze, M. Bode, M. Heide, G. Bihlmayer, S. Blügel, and R. Wiesendanger, Phys. Rev. Lett. 101, 027201 (2008).

${ }^{37}$ C. L. Gao, W. Wulfhekel, and J. Kirschner, Phys. Rev. Lett. 101, 267205 (2008).

${ }^{38}$ J. Hafner and D. Spišák, Phys. Rev. B 72, 144420 (2005).

${ }^{39}$ T. Moriya, Solid State Commun. 2, 239 (1964).

${ }^{40}$ C. Herring, Magnetism (Academic, New York, 1966).

${ }^{41}$ L. M. Sandratskii, Status Solidi B 136, 167 (1986).

${ }^{42}$ Y. Zhang and W. Yang, Phys. Rev. Lett. 80, 890 (1998).

${ }^{43}$ Y. Mokrousov, G. Bihlmayer, and S. Blügel, Phys. Rev. B 72, 045402 (2005).

${ }^{44}$ [www.flapw.de].

${ }^{45}$ P. Kurz, F. Förster, L. Nordström, G. Bihlmayer, and S. Blügel, Phys. Rev. B 69, 24415 (2004).
${ }^{46}$ W. Williams and J. L. Stanford, J. Magn. Magn. Mater. 1, 271 (1976).

${ }^{47}$ R. Skomski, Simple Models of Magnetism (Oxford University Press, Oxford, 2008).

${ }^{48}$ J. Slater, Phys. Rev. 36, 57 (1930).

${ }^{49}$ K. Terakura, N. Hamada, T. Oguchi, and T. Asada, J. Phys. F: Met. Phys. 12, 1661 (1982).

${ }^{50}$ A. Kubetzka, P. Ferriani, M. Bode, S. Heinze, G. Bihlmayer, K. von Bergmann, O. Pietzsch, S. Blugel, and R. Wiesendanger, Phys. Rev. Lett. 94, 087204 (2005).

${ }^{51}$ Y. Yayon, V. W. Brar, L. Senapati, S. C. Erwin, and M. F. Crommie, Phys. Rev. Lett. 99, 67202 (2007).

${ }^{52}$ C. Iacovita, M. V. Rastei, B. W. Heinrich, T. Brumme, J. Kortus, L. Limot, and J. P. Bucher, Phys. Rev. Lett. 101, 116602 (2008).

${ }^{53}$ C. L. Gao, W. Wulfhekel, and J. Kirschner, Phys. Rev. Lett. 101, 267205 (2008).

${ }^{54}$ M. Waśniowska, S. Schröder, P. Ferriani, and S. Heinze, Phys. Rev. B 82, 012402 (2010).

${ }^{55}$ D. Wortmann, S. Heinze, P. Kurz, G. Bihlmayer, and S. Blügel, Phys. Rev. Lett. 86, 4132 (2001).

${ }^{56}$ P. Ferriani, C. Lazo, and S. Heinze, Phys. Rev. B 82, 054411 (2010).

${ }^{57}$ S. Heinze, Appl. Phys. A 85, 407 (2006). 